\title{
Phylogenetic Position of Aquificales Based on the Whole Genome Sequences of Six Aquificales Species
}

\author{
Kenro Oshima, ${ }^{1}$ Yoko Chiba, ${ }^{2}$ Yasuo Igarashi, ${ }^{2}$ Hiroyuki Arai, ${ }^{2}$ and Masaharu Ishiii ${ }^{2}$ \\ ${ }^{1}$ Department of Agricultural and Environmental Biology, Graduate School of Agricultural and Life Sciences, \\ The University of Tokyo, Bunkyo-ku, Tokyo 113-8657, Japan \\ ${ }^{2}$ Department of Biotechnology, Graduate School of Agricultural and Life Sciences, The University of Tokyo, \\ Bunkyo-ku, Tokyo 113-8657, Japan \\ Correspondence should be addressed to Kenro Oshima, kenro@ims.u-tokyo.ac.jp
}

Received 10 February 2012; Revised 17 April 2012; Accepted 18 April 2012

Academic Editor: Hiromi Nishida

Copyright (๑) 2012 Kenro Oshima et al. This is an open access article distributed under the Creative Commons Attribution License, which permits unrestricted use, distribution, and reproduction in any medium, provided the original work is properly cited.

Species belonging to the order Aquificales are believed to be an early branching lineage within the Bacteria. However, the branching order of this group in single-gene phylogenetic trees is highly variable; for example, it has also been proposed that the Aquificales should be grouped with $\varepsilon$-proteobacteria. To investigate the phylogenetic position of Aquificales at the whole-genome level, here we reconstructed the phylogenetic trees of 18 bacteria including six Aquificales species based on the concatenated data of proteins shared by these bacteria. In the phylogenetic tree based on the whole-genome information, Aquificales was more closely related to Thermotogales than to Proteobacteria, suggesting that the Aquificales is a relatively early branching lineage within the Bacteria. Moreover, we classified the phylogenetic tree of each conserved orthologous protein by its topology. As a result, in the most major type of the phylogenetic trees, Aquificales was closely related to the Thermotogales. However, Aquificales was closely related to $\varepsilon$-proteobacteria in $21.0 \%$ of all phylogenetic trees, suggesting that many proteins phylogenetically related to the $\varepsilon$-proteobacteria may be encoded in the genomes of the members of the Aquificales. This unique feature may be responsible for the high variability in the branching order of Aquificales in single-gene phylogenetic trees.

\section{Introduction}

Species belonging to the order Aquificales are non-sporeforming, Gram-negative rods that are strictly thermophilic with optimal growth usually occurring above $65^{\circ} \mathrm{C}[1-3]$. In terms of metabolism, most species of Aquificales are hydrogen-oxidizing bacteria that utilize hydrogen as the sole electron donor and oxygen as the electron acceptor [4]. Alternatively, thiosulfate or sulfur can also be used as a source of energy. Because of their thermostability, many enzymes found in this group are of interest for industrial and biotechnological applications [5].

Presently, the Aquificales species are believed to be the earliest branching lineage within the Bacteria [6-10]. However, the branching order of this group in single-gene phylogenetic trees is highly variable, and the deep branching of Aquificales is not supported by many protein phylogenies. For example, Aquifex has been shown to be close to $\mathcal{E}$ proteobacteria $[11,12]$ or the Chlamydiae group [6] in many protein phylogenies. Conserved inserts and deletions in a number of different proteins also provide evidence that the Aquificales is a late branching group within the Bacteria [13]. Many of these analyses suggest that Aquificales might be more closely related to Proteobacteria than to Thermotogales. Therefore, it is important to understand the phylogenetic position of Aquificales within the bacterial phylogeny.

The phylogenetic tree based on 16S rRNA sequences provides the presently accepted framework for understanding the evolutionary relationships among bacteria [14]. However, phylogenetic analysis at the single-gene level may provide only a limited understanding of the relationships and evolutionary history of bacteria, especially the closely related species that diverged at almost the same time [15]. In addition, species phylogenies derived from comparisons of different genes do not always concur, which may be attributed to lateral gene transfer [16], saturation with respect to amino acid substitutions [17], or highly variable 
rates of evolution of individual genes [18, 19]. Therefore, it is believed that comparative studies based on the complete sequences of bacterial genomes should form the basis for phylogeny and, ultimately, taxonomy [20].

The phylogenies inferred from concatenated data of housekeeping proteins amplified the resolving power for delineating the phylogenetic relationships among prokaryotes [21-23]. The complete genome of Aquifex aeolicus was sequenced in 1998 [3], and the genomes of five Aquificales species (Hydrogenobacter thermophilus TK-6, Hydrogenobaculum sp. Y04AAS1, Persephonella marina, Sulfurihydrogenibium azorense and Sulfurihydrogenibium sp. YO3AOP1) have recently been sequenced $[24,25]$. Here we reconstructed the phylogenetic trees of 18 bacteria including six Aquificales bacteria based on the concatenated data of proteins shared by these bacteria. Moreover, the phylogenetic relationship between Aquificales and $\varepsilon$-proteobaceria was analyzed at the whole genome level.

\section{Materials and Methods}

In this study, we used 18 genome sequences from Aquifex aeolicus, Hydrogenobacter thermophilus TK-6, Hydrogenobaculum sp. Y04AAS1, Persephonella marina, Sulfurihydrogenibium azorense, Sulfurihydrogenibium sp. YO3AOP1, Bacillus subtilis subsp. subtilis str. 168, Burkholderia mallei ATCC 23344, Campylobacter jejuni subsp. jejuni NCTC 11168, Chlamydophila pneumoniae CWL029, Deinococcus radiodurans R1, Thermus thermophilus HB8, Escherichia coli str. K-12 substr. MG1655, Salmonella enterica subsp. enterica serovar Typhimurium LT2, Helicobacter pylori 26695, Pyrococcus horikoshii OT3, Thermotoga maritima MSB8 and Thermotoga petrophila RKU-1. These genome sequences was obtained from GenomeNet (http://www.genome.jp/).

First, BLASTP searches (each protein encoded in the genome of Hydrogenobacter thermophilus TK-6 was used as a query) were performed against 18 whole genomes by using stand-alone BLAST program [26]. If 18 different proteins from all 18 bacteria occupied the top 18 proteins of the result of the BLAST search, additional BLASTP searches were performed against 18 whole genomes by using each of top 18 proteins as a query. If the top 18 proteins in all 18 BLAST search are the same, we defined these 18 proteins as a conserved orthologous protein. This procedure enabled us to define 62 sets of orthologous proteins from the 18 genomes in our study. (see Supplementary Table 1 in supplementary material available online at doi:10.1155/2012/859264).

Next, we constructed 62 multiple-alignments using MUSCLE [27]. After that, a concatenated multiple alignment inferred from the 62 multiple alignments was generated. The concatenated alignment had 31,542 amino acid sites, including 15,442 gap/insertion sites that were not considered in this analysis. To avoid a potential cause for long branch attraction, we removed the most saturated sites from the whole multiple alignments according to the previously described method by Boussau et al. [28] as follows. First, PhyML [29] was used to build a starting phylogeny based on the whole multiple alignments, using the JTT model and a gamma law discretized in four classes to account for variation in the evolutionary rates. Second, to estimate how sites were modeled by the discretized gamma law, we plotted the distribution of expected relative evolutionary rates across sites as found by BppML (Supplementary Figure 1). Third, to reduce risks of long branch attraction, we decided to discard sites whose evolutionary rate was above the threshold of 2.0 (red line, Supplementary Figure 1). Finally, phylogenetic analyses were performed based on 10,000 amino acid sites. Based on the multiple alignments, a maximum likelihood (ML) tree was reconstructed using the PhyML [29] based on the JTT model and a gamma law discretized in four classes to account for variation in the evolutionary rates. Pyrococcus horikoshii was used as an outgroup. The confidence values (\%) were estimated with the bootstrap sampling method (200 replications).

In addition, to reduce the influence of compositional bias, we recoded the alignment without saturated sites in 4 states based on the physicochemical properties of the amino acids [28] as follows: aromatic (FWY) and hydrophobic (MILV) amino acids were grouped in a single state, basic amino-acids (HKR) in another, acidic (DENQ) amino acids in one more state, and the fourth state contained all other amino acids (AGPST) to the exception of cysteine which was coded as missing data. The ML tree was constructed with this recoded alignment by the GTR model, an estimated proportion of invariant sites, a gamma law discretized in 5 categories with its alpha parameter estimated, and 200 bootstrap replicates [28].

To construct the phylogenetic tree of six Aquificales species, two Thermotogales species, two $\gamma$-proteobacteria, and two $\varepsilon$-proteobacteria, we used 12 genome sequences from Aquifex aeolicus, Hydrogenobacter thermophilus TK6, Hydrogenobaculum sp. Y04AAS1, Persephonella marina, Sulfurihydrogenibium azorense, Sulfurihydrogenibium sp. YO3AOP1, Campylobacter jejuni subsp. jejuni NCTC 11168, Deinococcus radiodurans R1, Escherichia coli str. K-12 substr. MG1655, Salmonella enterica subsp. enterica serovar Typhimurium LT2, and Helicobacter pylori 26695.

To construct the phylogenetic tree of Thermales-Deinococcales species, Thermotogales species, $\gamma$-proteobacteria, and $\varepsilon$-proteobacteria, we used 8 genome sequences from Campylobacter jejuni subsp. jejuni NCTC 11168, Deinococcus radiodurans R1, Thermus thermophilus HB8, Escherichia coli str. K-12 substr. MG1655, Salmonella enterica subsp. enterica serovar Typhimurium LT2, Helicobacter pylori 26695, Thermotoga maritima MSB8, and Thermotoga petrophila RKU-1. The phylogenetic tree based on the concatenate data of the whole conserved orthologous proteins was constructed by the same method as described above. The ML trees of individual proteins were constructed using PhyML [28].

\section{Results and Discussion}

3.1. Phylogenetic Position of Aquificales Based on WholeGenome Sequences. First, we constructed ML trees based on the $16 \mathrm{~S}$ rRNA sequences of 18 bacteria (Figure 1(a)). This 


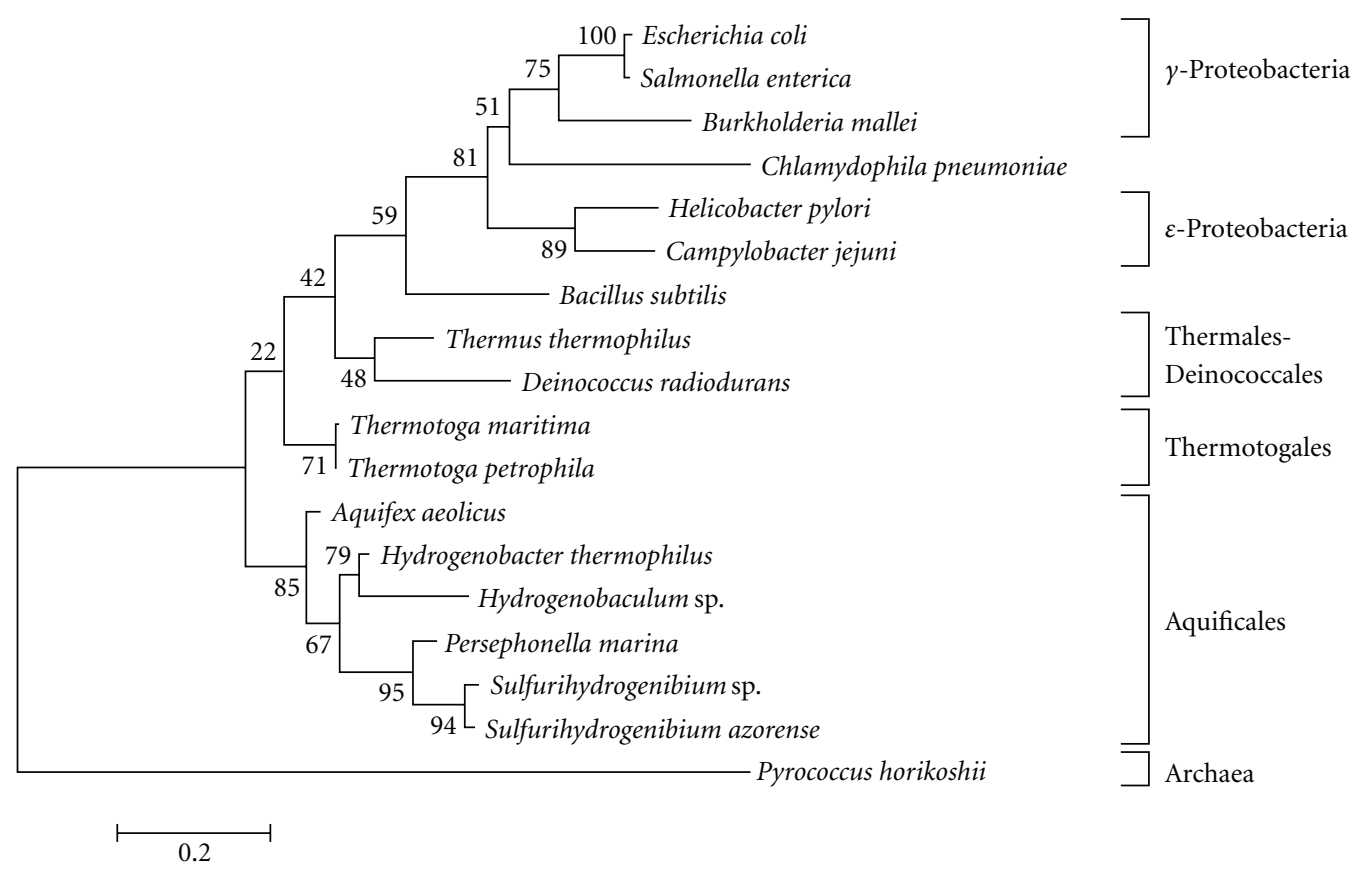

(a)

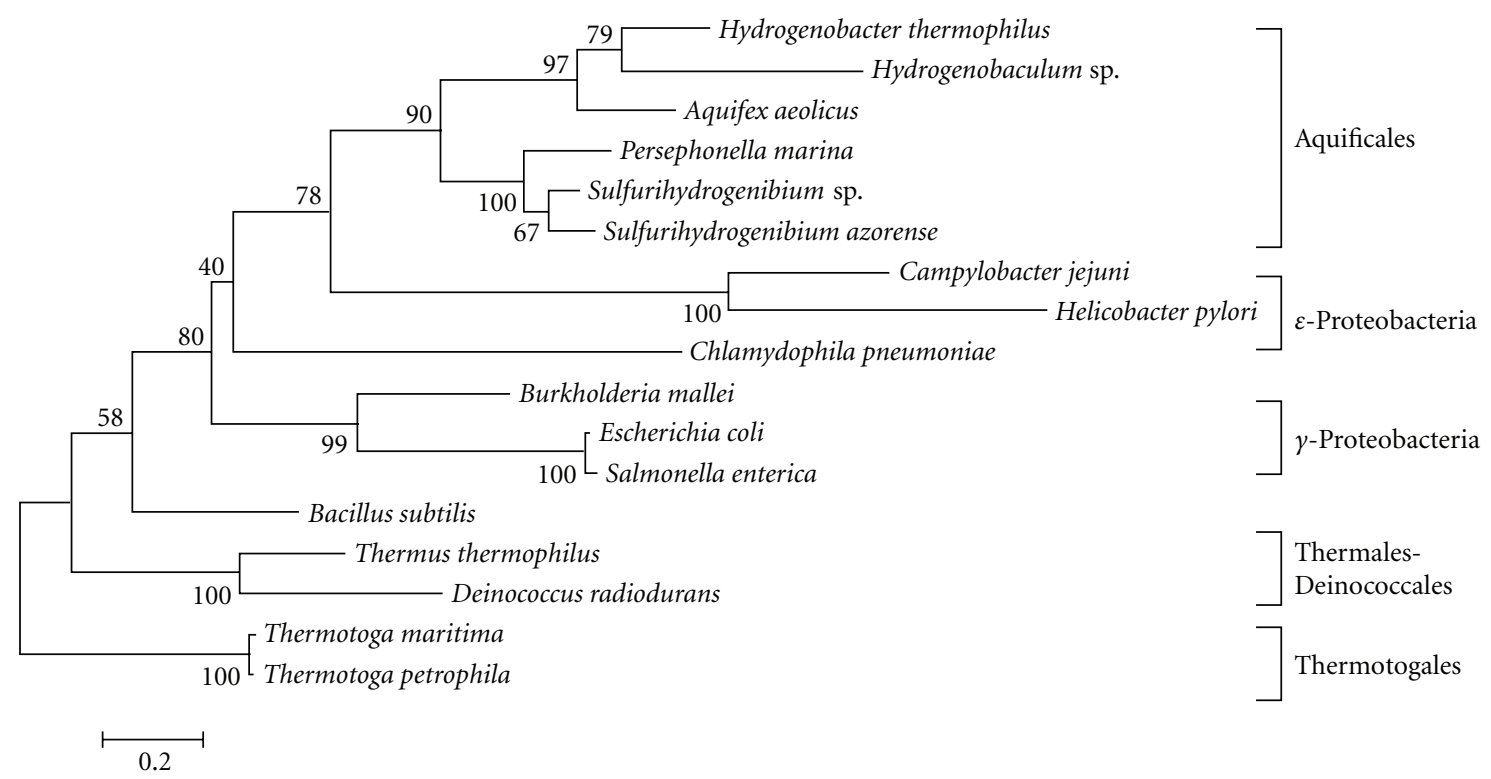

(b)

FIGURE 1: (a) Maximum likelihood tree based on the $16 \mathrm{~S}$ rRNA sequence comparison. The number at each node represents the percentage in the bootstrap analysis (1000 replicates). (b) Maximum likelihood tree based on the amino acid sequence of the transcription elongation factor. The number at each node represents the percentage in the bootstrap analysis (1000 replicates).

phylogenetic tree indicated that each bacteria belonging to Archaea, Aquificales, Thermales-Deinococcales, and Thermotogales was clustered as a clade. Thermales-Deinococcales was clustered with proteobacteria with $74 \%$ bootstrap support. The closest species to Archaea was the bacteria belonging to Thermotogales, and the second nearest neighbor was Aquificales, suggesting that the Aquificales species are an early branching lineage within the Bacteria. In contrast, the topology of the phylogenetic tree based on the amino acid sequences of transcription elongation factor (NusA) (Figure 1(b)) differed from that of the 16S rRNA gene. For example, Aquificales species were clustered with $\mathcal{\varepsilon}$ proteobacteria with $80 \%$ bootstrap support, suggesting that the Aquificales is a late branching group within the Bacteria.

To investigate the phylogenetic position of Aquificales species at the whole-genome level, we constructed a phylogenetic tree based on 18 whole genomes of Archaea, Aquificales, Thermales-Deinococcales, Thermotogales, and related 

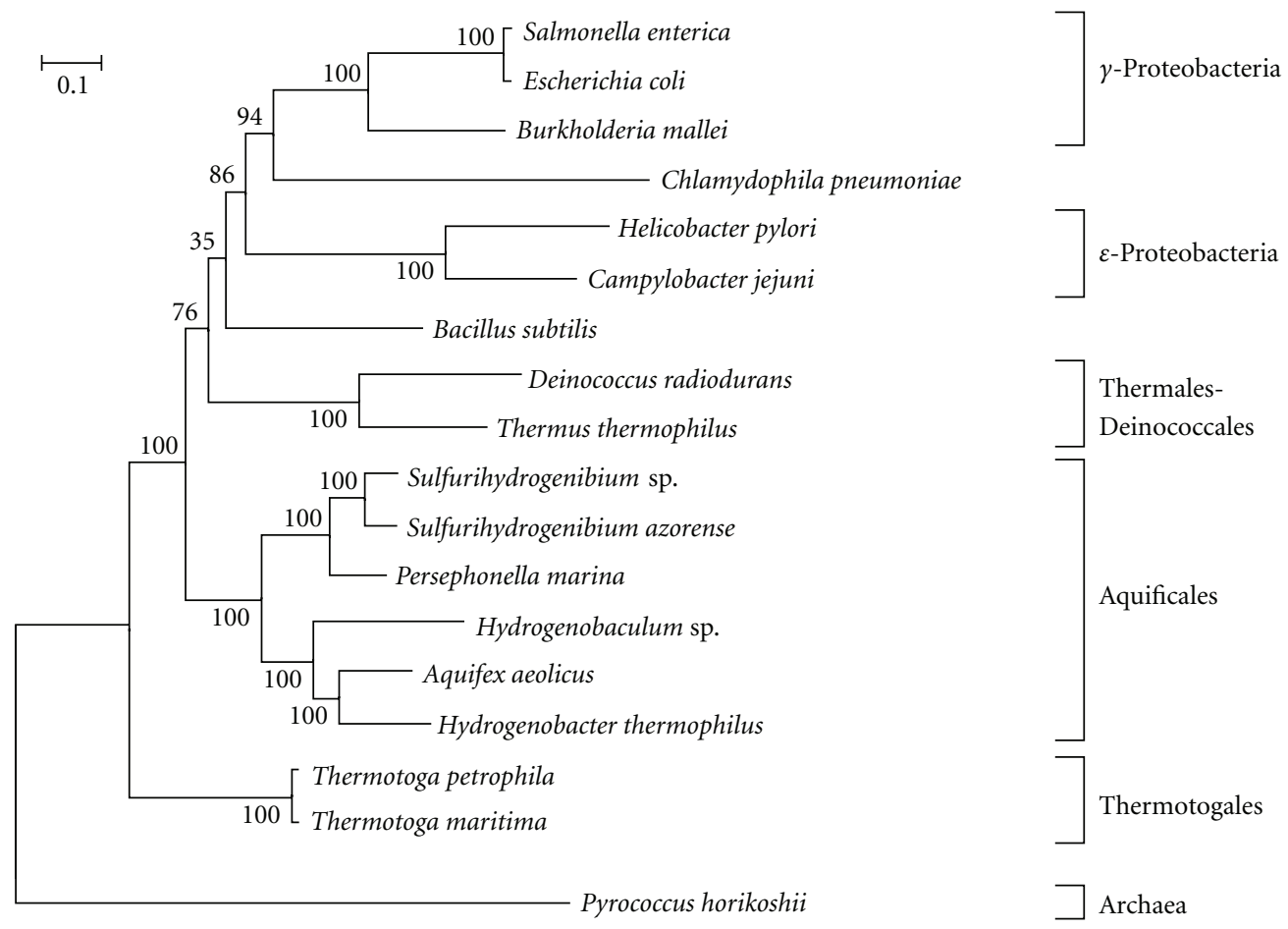

FIgURE 2: Maximum likelihood tree based on the comparison of 62 proteins; 10,000 amino acid sites were considered (see Section 2). The number at each node represents the percentage in the bootstrap analysis (200 replicates).

bacteria. First, 62 orthologous gene families that are shared by all 18 bacteria were selected (Supplementary Table 1). To avoid a potential cause for long branch attraction, we removed the most saturated sites from the whole multiple alignments according to the previously described method by Boussau et al. [28]. As a result, 10,000 amino acid sites were considered in the maximum likelihood analysis. The phylogenetic tree based on the whole-genome information indicated that the 18 bacteria were divided into six major groups (Archaea, Aquificales, Thermotogales, ThermalesDeinococcales, $\gamma$-proteobacteria, and $\varepsilon$-proteobacteria) with $100 \%$ bootstrap support. Analysis of signature sequences (consisting of conserved inserts or deletions) in highly conserved proteins suggested that the Aquificales diverged after the branching of Thermotogales, Thermales-Deinococcales, Cyanobacteria, Spirochetes, and Chlamydiae, but before the emergence of Proteobacteria [13]. However, in the phylogenetic tree based on the whole-genome information, the Archaea group was evolutionarily closely related to the Thermotogales, and Aquificales was a neighbor to Thermotogales with $76 \%$ bootstrap value (Figure 2 ). These analyses suggest that Aquificales is more closely related to Thermotogales than to Proteobacteria, which is consistent with the phylogenetic relationship showed by Boussau et al. [28]. To reduce the influence of compositional bias, we recoded the concatenated protein alignment in 4 states based on the physicochemical properties of the amino acids, and constructed a phylogenetic tree. As a result, although Bacillus subtilis was clustered with Thermales-Deinococcales, the
ML tree obtained by the recoded alignment (Supplemetary Figure 2) was very similar to the previous tree (Figure 2), implying that the Aquificales-Thermotogales grouping does not seem to result from compositional biases. These results suggest that the Aquificales species are a relatively early branching lineage within the Bacteria.

3.2. Phylogenetic Relationships between the Aquificales and $\varepsilon$-Proteobacteria. It has been proposed that the Aquificales should be grouped with the $\varepsilon$-proteobacteria [12], which is supported by the phylogenetic analysis of single protein sequences such as the transcription elongation factor (Figure 1(b)). However, the late branching of the Aquificales is not supported by the $16 \mathrm{~S}$ rRNA gene sequence tree (Figure 1(a)) and the phylogenetic tree based on the whole-genome information (Figure 2). To investigate the phylogenetic relationships between the Aquificales and $\varepsilon$ proteobacteria, we reconstructed phylogenetic trees of 12 bacteria including six Aquificales species, two Thermotogales species, two $\gamma$-proteobacteria, and two $\varepsilon$-proteobacteria based on the concatenated data of proteins shared by these bacteria. First, 271 orthologous gene families that are shared by all 12 bacteria were selected. As a result, 16,532 amino acid sites were considered in the ML analysis. The phylogenetic tree based on this whole-genome information indicated that the 12 bacteria were divided into four major groups (Aquificales, Thermotogales, $\gamma$-proteobacteria, and $\mathcal{E}$ proteobacteria) with $100 \%$ bootstrap support (Figure 3(a)). 


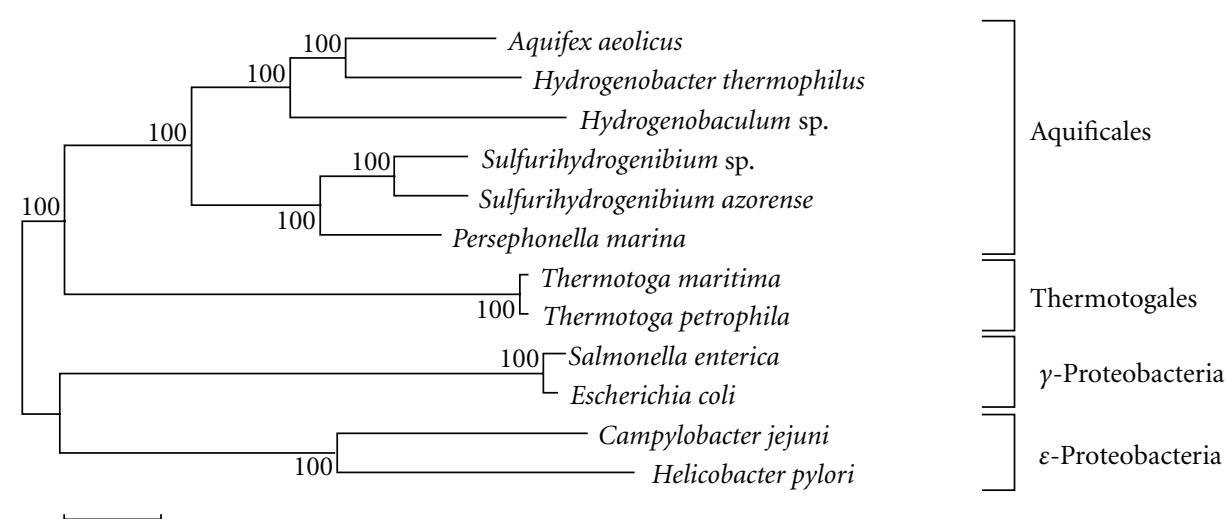

(a)
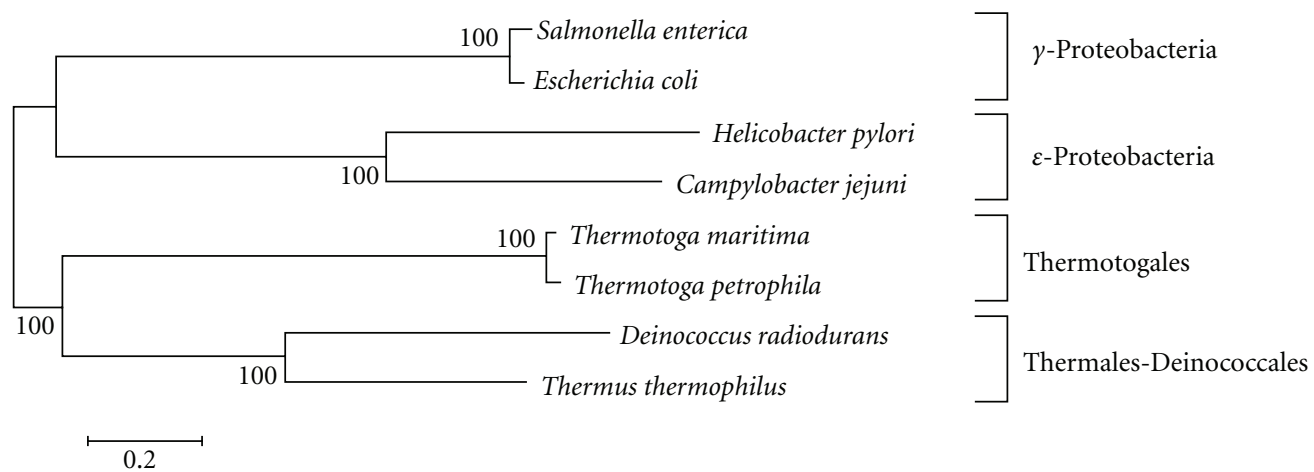

(b)

FigURE 3: Unrooted maximum likelihood tree based on whole-genome information by using (a) the 271 conserved proteins among Aquificales, Thermotogales, $\gamma$-proteobacteria and $\varepsilon$-proteobacteria, or (b) the 259 conserved proteins among Thermales, Thermotogales, $\gamma$-proteobacteria and $\varepsilon$-proteobacteria. The number at each node represents the percentage in the bootstrap analysis (200 replicates).

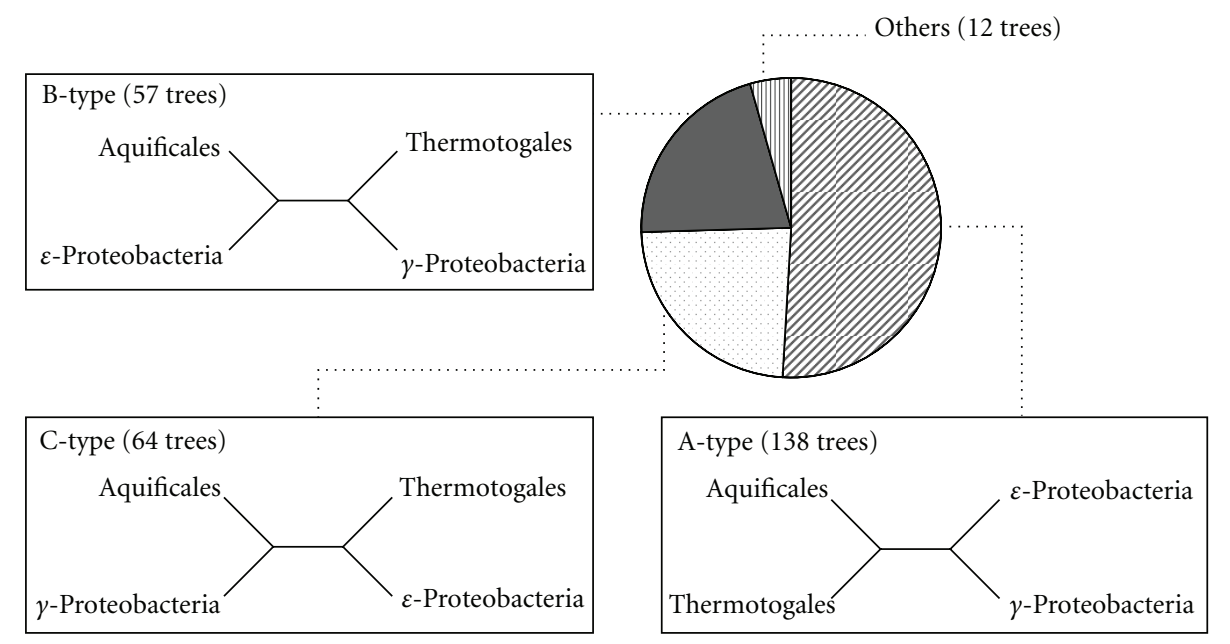

Figure 4: Distribution of topology of the phylogenetic trees of the 271 conserved proteins among Aquificales, Thermotogales, $\gamma$ proteobacteria, and $\varepsilon$-proteobacteria. 
TABLE 1: List of B-type conserved proteins that the Aquificales is clustered with $\varepsilon$-proteobacteria in the phylogenetic analysis (Figure 4). Accession numbers of conserved proteins of Hydrogenobacter thermophilus TK-6 are indicated.

\begin{tabular}{|c|c|}
\hline Accession number & Putative function \\
\hline YP_003431690 & transcription elongation factor \\
\hline YP_003432239 & ribosomal protein S9 \\
\hline YP_003432379 & ribosomal protein L18 \\
\hline YP_003432892 & 4-hydroxy-3-methylbut-2-enyl diphosphate reductase \\
\hline YP_003432936 & ATP-dependent protease \\
\hline YP_003433556 & UDP-N-acetylglucosamine pyrophosphorylase \\
\hline YP_003431738 & putative metalloprotease \\
\hline YP_003431749 & diaminopimelate decarboxylase \\
\hline YP_003431809 & dihydrodipicolinate reductase \\
\hline YP_003431998 & UDP-N-acetylglucosamine 1-carboxyvinyltransferase \\
\hline YP_003432481 & ribosomal protein $\mathrm{S} 20$ \\
\hline YP_003432953 & queuine tRNA-ribosyltransferase \\
\hline YP_003431834 & ATP-dependent protease La \\
\hline YP_003431839 & tRNA delta(2)-isopentenylpyrophosphate transferase \\
\hline YP_003431873 & 2-C-methyl-D-erythritol 4-phosphate cytidylyltransferase \\
\hline YP_003431915 & ribonuclease III \\
\hline YP_003432036 & riboflavin synthase alpha chain \\
\hline YP_003432044 & DNA polymerase I \\
\hline YP_003432149 & 2-methylthioadenine synthetase \\
\hline YP_003432165 & folylpolyglutamate synthase \\
\hline YP_003432232 & DNA polymerase III beta subunit \\
\hline YP_003432262 & $\begin{array}{l}\text { UDP-N-acetylglucosamine-N-acetylmuramyl- (pentapeptide) pyrophosphoryl-undecaprenol N- } \\
\text { acetylglucosamine transferase }\end{array}$ \\
\hline YP_003432385 & methionine aminopeptidase \\
\hline YP_003432408 & methionyl-tRNA synthetase \\
\hline YP_003432463 & fatty acid/phospholipid synthesis protein \\
\hline YP_003433015 & carboxyl-terminal protease \\
\hline YP_003433058 & rRNA methylase \\
\hline YP_003433377 & 3-phosphoshikimate 1-carboxyvinyltransferase \\
\hline YP_003433542 & arginyl-tRNA synthetase \\
\hline YP_003431843 & F0F1-type ATP synthase gamma subunit \\
\hline YP_003431889 & signal recognition particle GTPase \\
\hline YP_003432507 & ribosomal protein $\mathrm{L} 22$ \\
\hline YP_003432144 & Holliday junction resolvase \\
\hline YP_003432824 & DNA processing protein \\
\hline YP_003432257 & GTP-binding protein \\
\hline YP_003432274 & triosephosphate isomerase \\
\hline YP_003432330 & aspartate 1-decarboxylase \\
\hline YP_003432353 & uridylate kinase \\
\hline YP_003432374 & ribosomal protein L24 \\
\hline YP_003432380 & ribosomal protein S5 \\
\hline YP_003432524 & transcription antitermination protein \\
\hline YP_003432640 & methionyl-tRNA formyltransferase \\
\hline YP_003433333 & ribosomal protein L20 \\
\hline YP_003432384 & adenylate kinase \\
\hline YP_003432390 & ribosomal protein $\mathrm{S} 4$ \\
\hline YP_003432414 & thiol peroxidase \\
\hline
\end{tabular}


TABle 1: Continued.

\begin{tabular}{ll}
\hline Accession number & Putative function \\
\hline YP_003432533 & orotidine 5' -phosphate decarboxylase \\
YP_003432615 & S-adenosyl-methyltransferase \\
YP_003432911 & carbamoyl-phosphate synthase small subunit \\
YP_003432886 & dihydrodipicolinate synthase \\
YP_003432967 & membrane protein \\
YP_003432968 & GMP synthase \\
YP_003433028 & hypothetical protein HTH_1376 \\
YP_003433124 & homoserine kinase \\
YP_003433221 & UDP-glucose-4-epimerase \\
YP_003433380 & pantothenate metabolism flavoprotein \\
YP_003433549 & cell cycle protein \\
\hline
\end{tabular}

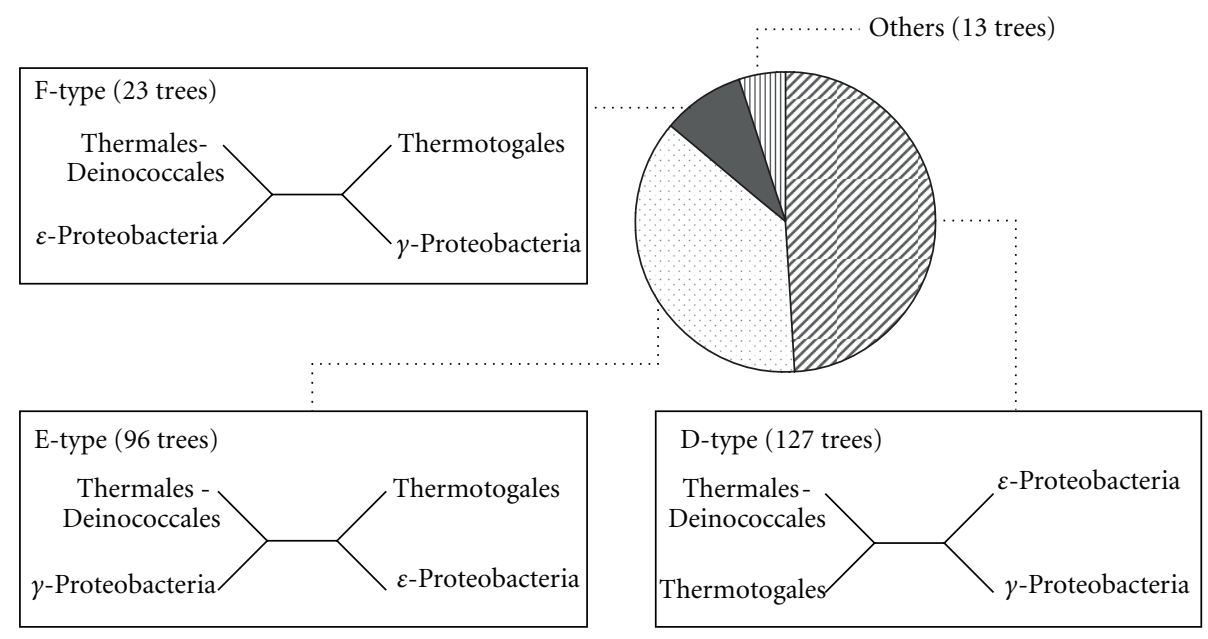

FIGURE 5: Distribution of topology of the phylogenetic trees of the 259 conserved proteins among Thermales, Thermotogales, $\gamma$ proteobacteria and $\varepsilon$-proteobacteria.

In addition, the phylogenetic tree based on the wholegenome information indicated that the Aquificales group was clustered with the Thermotogales group with 100\% bootstrap support (Figure 3(a)).

Next, to investigate the contribution of each protein to the whole-genome phylogenetic tree, we constructed $271 \mathrm{ML}$ trees from 271 protein sets. We classified these trees into the following three types (Figure 4): A-type, the Aquificales group is more closely related to the Thermotogales group; B-type, the Aquificales group is more closely related to the $\varepsilon$-proteobacteria group; $\mathrm{C}$-type, the Aquificales group is more closely related to the $\gamma$-proteobacteria group. The most frequent type of these phylogenetic trees was A-type (138 trees), which is consistent with the results obtained from the phylogenetic tree based on the 271 conserved proteins (Figure 3(a)). Interestingly, B-type trees occupied $21.0 \%$ (57 trees) of all phylogenetic trees (Table 1). For example, the Aquificales was clustered with the $\mathcal{E}$-proteobacteria with $94 \%$ bootstrap support in the phylogenetic tree of DNA polymerase I (Supplementary Figure 3). These results suggest that many proteins phylogenetically related to the $\varepsilon$-proteobacteria may be encoded in the genomes of the members of the Aquificales order.

To compare this profile with that of other bacteria, we performed the same phylogenetic analysis against 259 conserved proteins among Thermales-Deinococcales, Thermotogales, $\gamma$-proteobacteria, and $\varepsilon$-proteobacteria. As a result, the Thermales-Deinococcales group was clustered with the Thermotogales group with $100 \%$ bootstrap support in the phylogenetic tree based on the whole genome conserved proteins (Figure 3(b)). Next, we classified the phylogenetic tree of each protein into the following three types (Figure 5); D-type, the Thermales-Deinococcales group is more closely related to the Thermotogales group; E-type, the ThermalesDeinococcales group is more closely related to the $\gamma$ proteobacteria group; F-type, the Thermales-Deinococcales group is more closely related to the $\varepsilon$-proteobacteria group. The most frequent type of these phylogenetic trees was D-type (127 trees), and E-type trees occupied 37.1\% (96 trees) of all phylogenetic trees (Figure 5). In contrast, Ftype trees occupied only $8.9 \%$ (23 trees) of all phylogenetic trees (Figure 5), suggesting that the phylogenetic relationship 
between the Thermales-Deinococcales and $\varepsilon$-proteobacteria may be low compared to the Aquificales.

These results support the hypothesis that many proteins phylogenetically close to the $\mathcal{\varepsilon}$-proteobacteria may be encoded in the genomes of the Aquificales. This unique feature may be responsible for the high variability in the branching order of Aquificales in single-gene phylogenetic trees. Moreover, these results raised the possibility that a large horizontal gene transfer had occurred between the Aquificales and $\mathcal{E}$-proteobacteria, which was suggested by Boussau et al. [28]. This hypothesis might be supported by the fact that $\varepsilon$-proteobacteria include hydrogen-oxidizing bacteria and sulfur-oxidizing bacteria [30] which occupy the same ecological niche with Aquificales.

Several house-keeping proteins have often been used for the phylogenetic analyses of bacteria [31-33]. However, our results suggest that the phylogenetic position of single proteins is highly variable even for transcription elongation factor and DNA polymerase I. Therefore, whole-genome level phylogenetic approaches are extremely important and will possibly play a crucial role in the future studies of microbial evolution.

\section{Acknowledgments}

The authors thank anonymous reviewer for valuable comments. This work was supported in part by a Grant-inAid for scientific research (A) (21248010) from the Japan Society for the Promotion of Science (JSPS), a Grant-in-Aid for JSPS fellows (23-3030), and a funding program for Next Generation World-Leading Researchers (GS005).

\section{References}

[1] W. Eder and R. Huber, "New isolates and physiological properties of the Aquificales and description of Thermocrinis albus sp. nov," Extremophiles, vol. 6, no. 4, pp. 309-318, 2002.

[2] T. Kawasumi, Y. Igarashi, T. Kodama, and Y. Minoda, "Hydrogenobacter thermophilus gen. nov., sp. nov., an extremely thermophilic, aerobic, hydrogen-oxidizing bacterium," International Journal of Systematic Bacteriology, vol. 34, no. 1, pp. 5-10, 1984.

[3] G. Deckert, P. V. Warren, T. Gaasterland et al., "The complete genome of the hyperthermophilic bacterium Aquifex aeolicus," Nature, vol. 392, no. 6674, pp. 353-358, 1998.

[4] R. Huber and W. Eder, "Aquificales," in The Prokaryotes: An Evolving Electronic Resource for the Microbiological Community. Release 3.8, M. Dworkin, S. Falkow, E. Rosenberg, K.-H. Schleifer, and E. Stackebrandt, Eds., Springer, New York, NY, USA, 2002.

[5] H. Huber and K. O. Stetter, "Hyperthermophiles and their possible potential in biotechnology," Journal of Biotechnology, vol. 64, no. 1, pp. 39-52, 1998.

[6] S. Burggraf, G. J. Olsen, K. O. Stetter, and C. R. Woese, "A phylogenetic analysis of Aquifex pyrophilus," Systematic and Applied Microbiology, vol. 15, no. 3, pp. 352-356, 1992.

[7] G. J. Olsen, C. R. Woese, and R. Overbeek, "The winds of (evolutionary) change: breathing new life into microbiology," Journal of Bacteriology, vol. 176, no. 1, pp. 1-6, 1994.
[8] M. Bocchetta, S. Gribaldo, A. Sanangelantoni, and P. Cammarano, "Phylogenetic depth of the bacterial genera Aquifex and Thermotoga inferred from analysis of ribosomal protein, elongation factor, and RNA polymerase subunit sequences," Journal of Molecular Evolution, vol. 50, no. 4, pp. 366-380, 2000.

[9] J. R. Brown and W. F. Doolittle, "Root of the universal tree of life based on ancient aminoacyl-tRNA synthetase gene duplications," Proceedings of the National Academy of Sciences of the United States of America, vol. 92, no. 7, pp. 2441-2445, 1995.

[10] C. Pitulle, Y. Yang, M. Marchiani et al., "Phylogenetic position of the genus Hydrogenobacter," International Journal of Systematic Bacteriology, vol. 44, no. 4, pp. 620-626, 1994.

[11] E. J. Deeds, H. Hennessey, and E. I. Shakhnovich, "Prokaryotic phylogenies inferred from protein structural domains," Genome Research, vol. 15, no. 3, pp. 393-402, 2005.

[12] T. Cavalier-Smith, "The neomuran origin of archaebacteria, the negibacterial root of the universal tree and bacterial megaclassification," International Journal of Systematic and Evolutionary Microbiology, vol. 52, no. 1, pp. 7-76, 2002.

[13] E. Griffiths and R. S. Gupta, "Signature sequences in diverse proteins provide evidence for the late divergence of the Order Aquificales," International Microbiology, vol. 7, no. 1, pp. 4152,2004

[14] C. R. Woese, O. Kandler, and M. L. Wheelis, "Towards a natural system of organisms: proposal for the domains Archaea, Bacteria, and Eucarya," Proceedings of the National Academy of Sciences of the United States of America, vol. 87, no. 12, pp. 4576-4579, 1990.

[15] G. E. Fox, J. D. Wisotzkey, and P. Jurtshuk, "How close is close: 16S rRNA sequence identity may not be sufficient to guarantee species identity," International Journal of Systematic Bacteriology, vol. 42, no. 1, pp. 166-170, 1992.

[16] P. Forterre and H. Philippe, "Where is the root of the universal tree of life?” BioEssays, vol. 21, no. 10, pp. 871-879, 1999.

[17] W. F. Doolittle, "Phylogenetic classification and the universal tree," Science, vol. 284, no. 5423, pp. 2124-2128, 1999.

[18] M. Hasegawa, H. Kishino, and T. A. Yano, "Estimation of branching dates among primates by molecular clocks of nuclear DNA which slowed down in Hominoidea," Journal of Human Evolution, vol. 18, no. 5, pp. 461-476, 1989.

[19] M. A. Huynen and P. Bork, "Measuring genome evolution," Proceedings of the National Academy of Sciences of the United States of America, vol. 95, no. 11, pp. 5849-5856, 1998.

[20] S. Razin, D. Yogev, and Y. Naot, "Molecular biology and pathogenicity of mycoplasmas," Microbiology and Molecular Biology Reviews, vol. 62, no. 4, pp. 1094-1156, 1998.

[21] J. R. Brown, C. J. Douady, M. J. Italia, W. E. Marshall, and M. J. Stanhope, "Universal trees based on large combined protein sequence data sets," Nature Genetics, vol. 28, no. 3, pp. 281$285,2001$.

[22] K. Oshima and H. Nishida, "Phylogenetic relationships among mycoplasmas based on the whole genomic information," Journal of Molecular Evolution, vol. 65, no. 3, pp. 249-258, 2007.

[23] K. Oshima and H. Nishida, "Detection of the genes evolving under Ureaplasma selection," Journal of Molecular Evolution, vol. 66, no. 5, pp. 529-532, 2008.

[24] H. Arai, H. Kanbe, M. Ishii, and Y. Igarashi, "Complete genome sequence of the thermophilic, obligately chemolithoautotrophic hydrogen-oxidizing bacterium Hydrogenobacter thermophilus TK-6," Journal of Bacteriology, vol. 192, no. 10, pp. 2651-2652, 2010.

[25] A. L. Reysenbach, N. Hamamura, M. Podar et al., "Complete and draft genome sequences of six members of the Aquificales," 
Journal of Bacteriology, vol. 191, no. 6, pp. 1992-1993, 2009.

[26] S. F. Altschul, W. Gish, W. Miller, E. W. Myers, and D. J. Lipman, "Basic local alignment search tool," Journal of Molecular Biology, vol. 215, no. 3, pp. 403-410, 1990.

[27] R. C. Edgar, "MUSCLE: multiple sequence alignment with high accuracy and high throughput," Nucleic Acids Research, vol. 32, no. 5, pp. 1792-1797, 2004.

[28] B. Boussau, L. Guéguen, and M. Gouy, "Accounting for horizontal gene transfers explains conflicting hypotheses regarding the position of aquificales in the phylogeny of Bacteria," $B M C$ Evolutionary Biology, vol. 8, no. 1, p. 272, 2008.

[29] S. Guindon, J. F. Dufayard, V. Lefort, M. Anisimova, W. Hordijk, and O. Gascuel, "New algorithms and methods to estimate maximum-likelihood phylogenies: assessing the performance of PhyML 3.0," Systematic Biology, vol. 59, no. 3, pp. 307-321, 2010.

[30] J. W. Voordeckers, M. H. Do, M. Hügler, V. Ko, S. M. Sievert, and C. Vetriani, "Culture dependent and independent analyses of 16S rRNA and ATP citrate lyase genes: a comparison of microbial communities from different black smoker chimneys on the Mid-Atlantic Ridge," Extremophiles, vol. 12, no. 5, pp. 627-640, 2008.

[31] W. Ludwig, O. Strunk, S. Klugbauer et al., "Bacterial phylogeny based on comparative sequence analysis," Electrophoresis, vol. 19, no. 4, pp. 554-568, 1998.

[32] M. Falah and R. S. Gupta, "Phylogenetic analysis of mycoplasmas based on Hsp70 sequences: cloning of the dnaK (hsp70) gene region of Mycoplasma capricolum," International Journal of Systematic Bacteriology, vol. 47, no. 1, pp. 38-45, 1997.

[33] V. Kamla, B. Henrich, and U. Hadding, "Phylogeny based on elongation factor Tu reflects the phenotypic features of mycoplasmas better than that based on 16S rRNA," Gene, vol. 171, no. 1, pp. 83-87, 1996. 

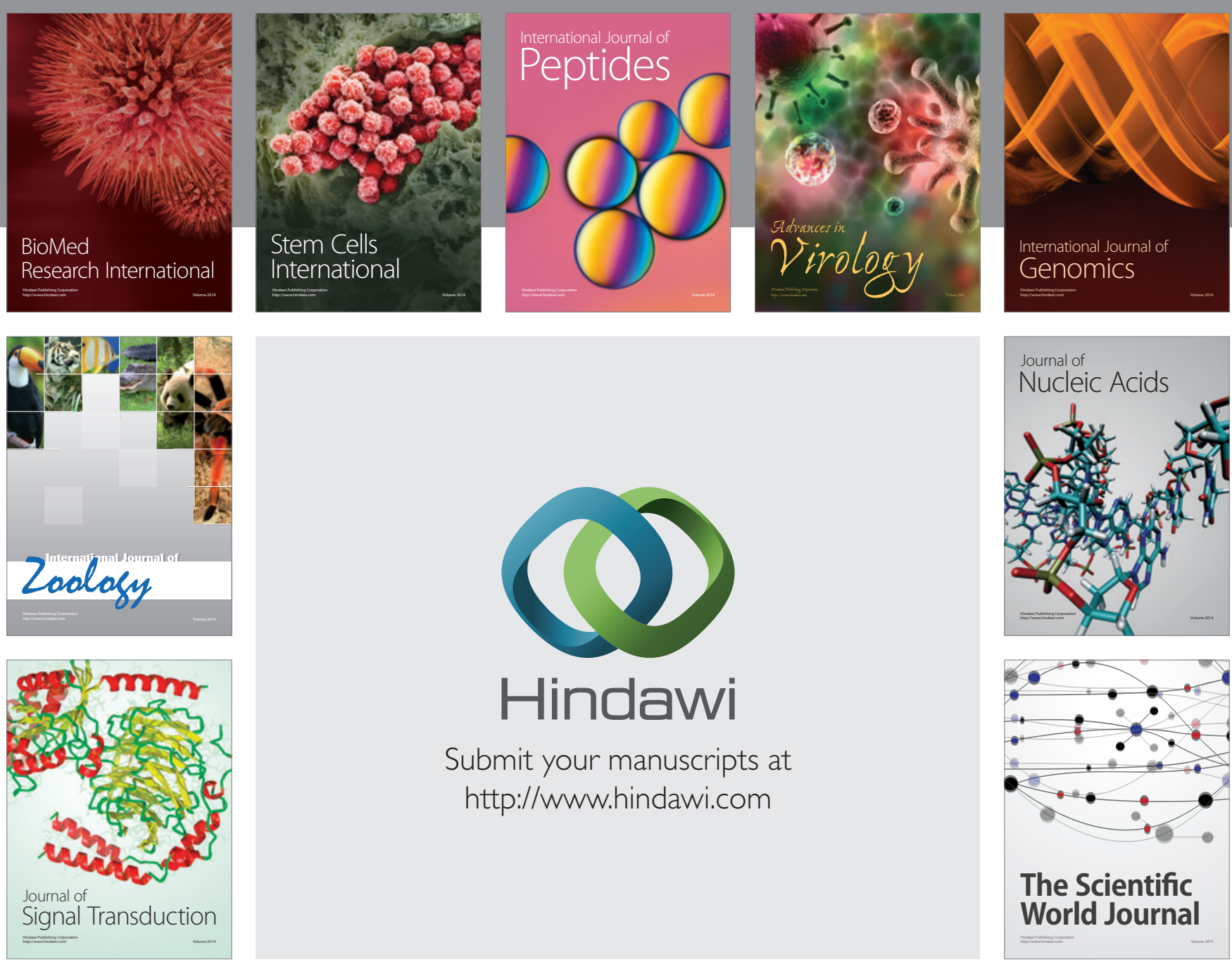

Submit your manuscripts at

http://www.hindawi.com
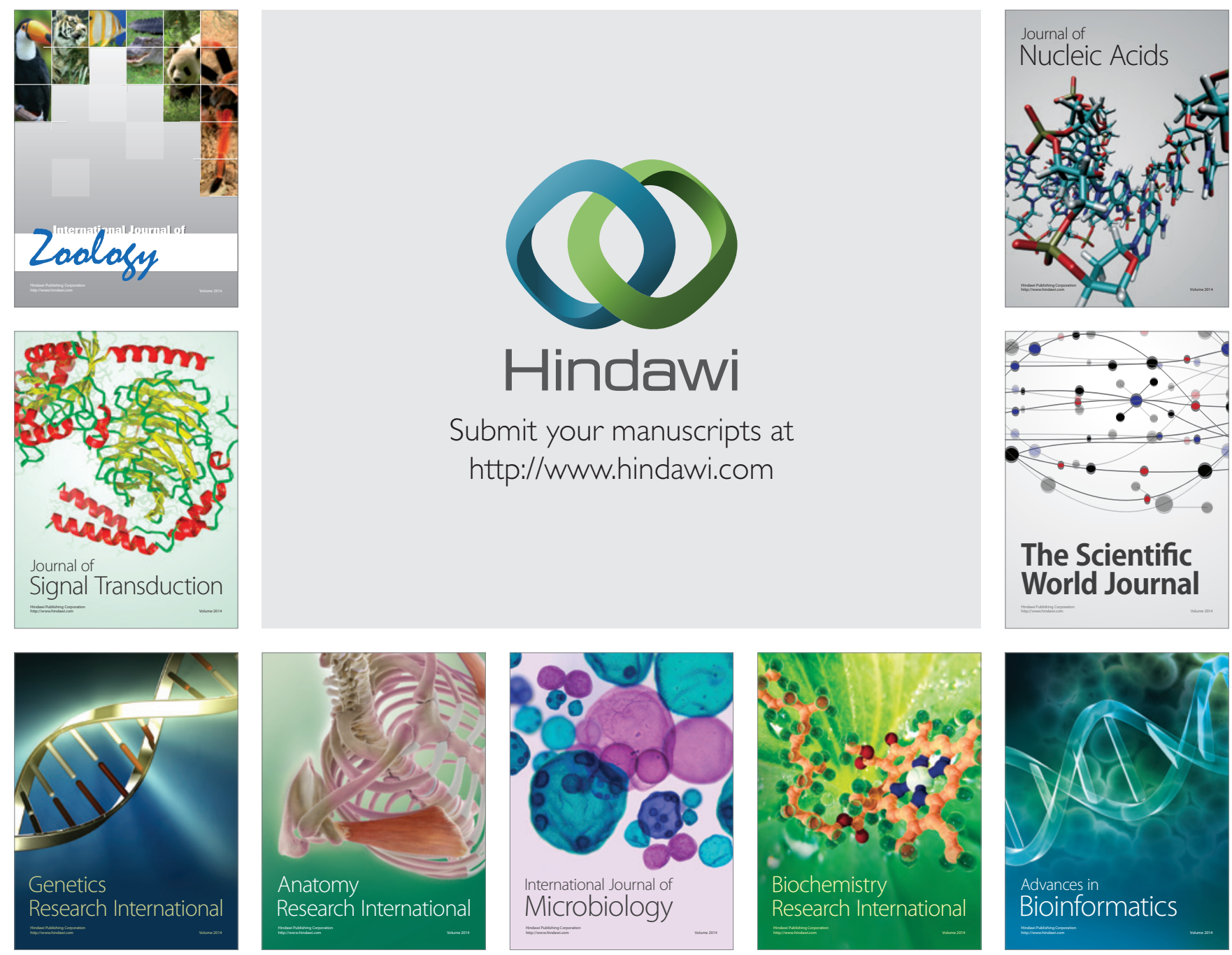

The Scientific World Journal
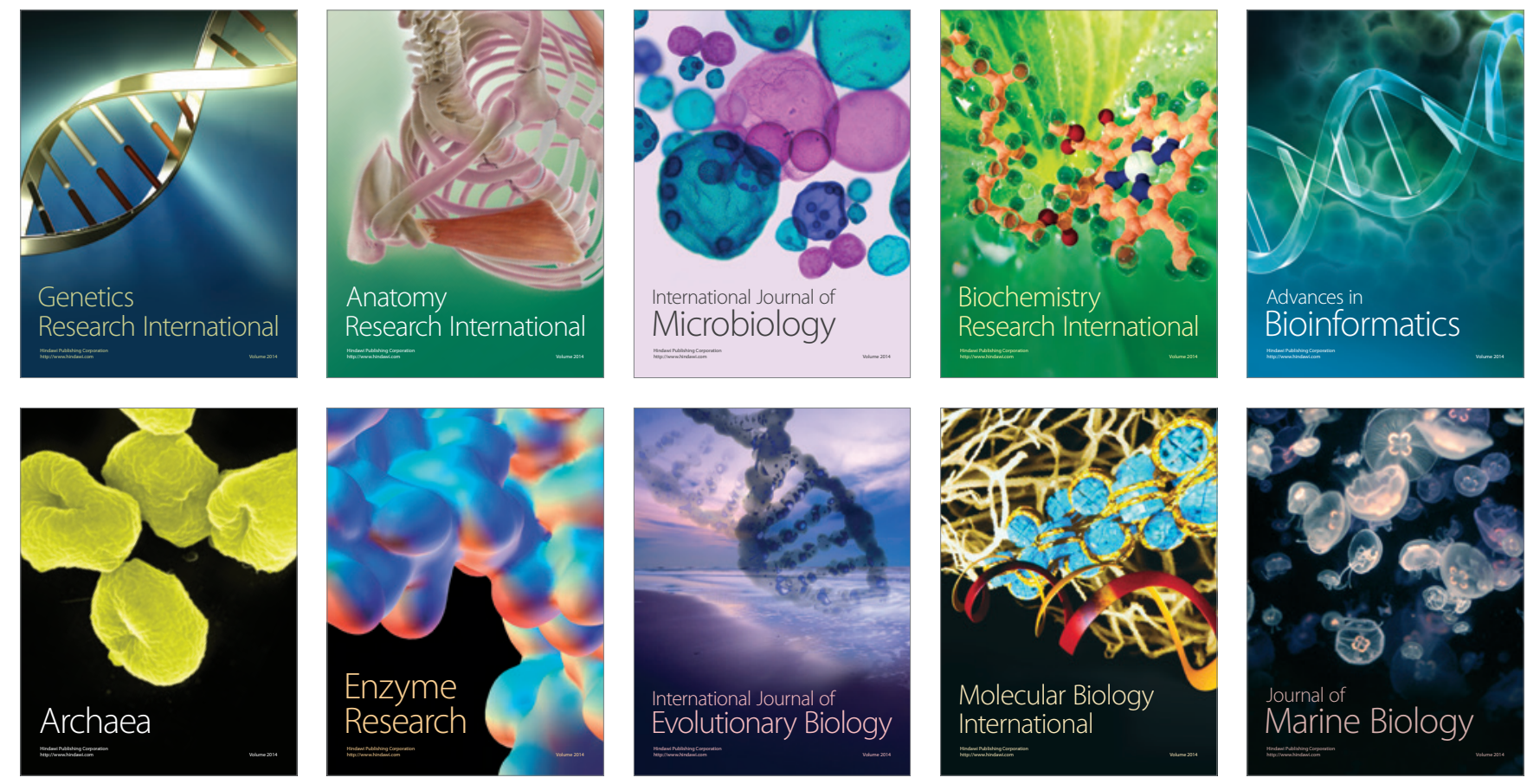